\title{
What is the functional relevance of prefrontal cortex entrainment to hippocampal theta rhythms?
}

Edited by:

Bernard W. Balleine, University of

California Los Angeles, USA

Reviewed by:

Matthew W. Jones, University of Bristol, UK

Bernard W. Balleine, University of California Los Angeles, USA

*Correspondence:

James M. Hyman received a B.S. in psychology from Virginia Commonwealth University in 1998, and a Ph.D. in psychology from Boston University. My graduate work was completed in Dr. Michael Hasselmo's lab, and it was there I learned the techniques to acquire and analyze in vivo electrophysiological data from behaving animals, and developed my skills for designing creative behavioral and analytical approaches to understand how activity in multiple neural areas related to the cognitive processes of behavior. I'm currently a postdoctoral fellow with Dr. Jeremy Seamans, which has allowed me to increase my skill-set to encompass detailed knowledge of the prefrontal cortex and advanced mathematical analysis techniques of neuronal populations. My current experimental focus is on the role of prefrontal cortex activity in cognition and mental disorders.

hyman.jm@gmail.com

\section{James Michael Hyman ${ }^{1}$, Michael Erik Hasselmo ${ }^{2}$ and Jeremy Keith Seamans ${ }^{1}$}

1 Brain Research Centre, Department of Psychiatry, University of British Columbia, Vancouver, BC, Canada

2 Center for Memory and Brain, Department of Psychology, Boston University, Boston, MA, USA

There has been considerable interest in the importance of oscillations in the brain and in how these oscillations relate to the firing of single neurons. Recently a number of studies have shown that the spiking of individual neurons in the medial prefrontal cortex (mPFC) become entrained to the hippocampal (HPC) theta rhythm. We recently showed that theta-entrained mPFC cells lost theta-entrainment specifically on error trials even though the firing rates of these cells did not change (Hyman et al., 2010). This implied that the level of HPC theta-entrainment of mPFC units was more predictive of trial outcome than differences in firing rates and that there is more information encoded by the mPFC on working memory tasks than can be accounted for by a simple rate code. Nevertheless, the functional meaning of mPFC entrainment to HPC theta remains a mystery. It is also unclear as to whether there are any differences in the nature of the information encoded by theta-entrained and non-entrained mPFC cells. In this review we discuss mPFC entrainment to HPC theta within the context of previous results as well as provide a more detailed analysis of the Hyman et al. (2010) data set. This re-analysis revealed that theta-entrained mPFC cells selectively encoded a variety of task-relevant behaviors and stimuli while never theta-entrained mPFC cells were most strongly attuned to errors or the lack of expected rewards. In fact, these error responsive neurons were responsible for the error representations exhibited by the entire ensemble of mPFC neurons. A theta reset was also detected in the post-error period. While it is becoming increasingly evident that mPFC neurons exhibit correlates to virtually all cues and behaviors, perhaps phase-locking directs attention to the task-relevant representations required to solve a spatially based working memory task while the loss of theta-entrainment at the start of error trials may represent a shift of attention away from these representations. The subsequent theta reset following error commission, when coupled with the robust responses of never theta-entrained cells, could produce a potent error-evoked signal used to alert the rat to changes in the relationship between task-relevant cues and reward expectations.

Keywords: prefrontal cortex, hippocampus, theta rhythm, working memory, oscillations

\section{INTRODUCTION}

Traditionally the encoding of information by neural networks was believed to be reflected mainly as changes in the firing rate of neurons. In the case of the medial prefrontal cortex (mPFC) increases in firing rates encode information about cues, responses, and task general rules as well as maintaining an active representation of recently 
Specific task-relevant information is not transferred from the

hippocampus to prefrontal units via theta-entrainment

While theta-entrained prefrontal units do encode task-relevant information more than never theta-entrained cells, there are no differences in firing rates when theta-entrainment is lost on incorrect trials. Indicating that prefrontal cells robustly represent task-relevant information on their own and that theta-entrainment exerts independent effects.

Prefrontal cortex-HPC theta interactions on correct trials Previous studies have shown that prefrontal ensembles contain egocentric representations of the rat's current experience, while hippocampal ensembles represent allocentric information about the state of the world. Working memory task require the successful integration of these two representations, which likely occurs via theta interactions because only theta interactions are impaired on incorrect trials while the representations in each area appear unaffected.

Prefrontal unit post-error commission discharges

A group of mPFC neurons that were never theta-entrained had a significant increase in firing rates $\sim 400 \mathrm{~ms}$ after an error response. These same cells were also more selective for error trials than theta-entrained cells. When these cells are included in $\mathrm{mPFC}$ ensemble activity state analysis there is significantly more separation between correct and error responses than when they are not included.

Hippocampal theta phase reset after errors

Following error responses the HPC theta rhythm resets and clear theta oscillations are visible in the averaged LFP. This indicates that errors lead to a change in HPC processing states that may ensure increased plastic changes involving cells representing the lack of reinforcement following an error.

Rodent error-related negativity The post-error increased firing rates of some mPFC neurons and the reset of HPC theta rhythm may be indicative of a rodent ERN. Both post-error medial mPFC discharge bursts and theta phase resets are theorized to generate the ERN observed in human scalp EEG recordings. The current findings provide tentative support for both mechanisms in the rodent brain. acquired information (for review see Miller et al., 2002). Recently there has been a growing interest in the idea that information may be processed by $\mathrm{mPFC}$ neurons through phase-locking to field oscillations, especially to hippocampal (HPC) theta oscillations (Hyman et al., 2005; Siapas et al., 2005). Hyman et al. (2010) found that functional synchrony between the HPC and the mPFC at theta frequencies during the sample phase of a working memory task was much more predictive of trial outcomes than stimulus or action driven discharge changes of mPFC units. Indeed it was only when phase-locking was lost that performance suffered. However, not all cells phase-locked to HPC theta and the functional differences in phase-locked vs. non-phase-locked mPFC cells is currently unclear.

Here we will review the relevant literature on mPFC phase-locking to HPC theta rhythms and will perform a re-analysis of Hyman et al. (2010) in order to gain insight into the nature of the processing that occurs in $\mathrm{mPFC}$ during periods of strong vs. weak theta-entrainment. We found that firing rate changes can encode task-relevant behaviors and stimuli equally well in the presence or absence of HPC theta-entrainment, suggesting that theta-entrainment may not be a means to transfer specific information to mPFC neurons. Rather entrainment may serve to focus attention on the $\mathrm{mPFC}$ representations that are relevant for the performance of spatially based memory tasks. In contrast, during periods of weak thetaentrainment a different processing state may arise within the $\mathrm{mPFC}$, one that is dominated by the activity of a unique population of never theta-entrained neurons that are highly selective for error responses. In this way, even though the $\mathrm{mPFC}$ processes and represents a vast array of stimuli and behaviors, entrainment to various rhythms such as theta may help focus attention on a limited set of these.

\section{MATERIALS AND METHODS ENCODING BY mPFC SINGLE-UNITS WITH AND WITHOUT HPC THETA-ENTRAINMENT}

We re-analyzed the data from Hyman et al. (2010) to see if there were any differences in information encoding between cells with periods of thetaentrainment (theta cells) and those that had no theta-entrainment during the entire recording session ("never theta" cells). The previous analysis of firing activity in Hyman et al. (2010) used Kruskal-Wallis non-parametric ANOVAs to compare firing rates between error and correct trials for each trial phase and found that practically all theta-entrained cells had similar firing rates irrespective of trial outcome. For the current analysis we examined the degree of selectivity in firing rates between different behavioral epochs of interest. A selectivity index for each unit $i$ with respect to the type of behavior [sample or test lever press (LP)] or trial outcome (correct or error) was obtained by grouping the firing rates into two classes $(\mathrm{A}, \mathrm{B})$ corresponding to the examined behavioral conditions, and computing:

$$
d_{i}^{\prime}=\frac{\left|\left\langle\left\{r_{i}(t) \mid t \in A\right\}\right\rangle-\left\langle\left\{r_{i}(t) \mid t \in B\right\}\right\rangle\right|}{\sqrt{\sigma_{i, t \in A}^{2}+\sigma_{i, t \in B}^{2}}}
$$

where $\langle\bullet\rangle$ denotes the mean. The sets A and B refer to the two behavioral conditions compared: trial specific behaviors (correct or error trial sample or test LP vs. baseline ITI), trial specific lever locations (both task phase correct trial right LP's vs. all correct trial left LP's), and task general information (correct trial sample LP's vs. correct trial test LP's). Firing rates for the time bins defining these sets were collected from the 2-s periods around the relevant behaviors.

\section{SELECTIVITY INDICES FOR “NEVER THETA" NEURONS}

To examine how theta and "never theta" cells responded to error commission we computed selectivity indices by comparing activity during the 1-s periods before and after test LP's (cells with mean firing rates $<0.1 \mathrm{~Hz}$ for either correct or error periods were excluded; $22 / 74$ cells). This was done for both correct and error trials. Since erroneous LP's were made on a different lever than correct responses for the same trial type, comparisons were made to correct trial LP's from the opposite trial type to ensure that any differences were not due to spatial location alone. Selectivity index values were initially compared with a twofactor ANOVA (cell type, trial outcome) and then $t$-tests and Wilcoxon rank sum tests were used for more direct comparisons. Note that selectivity indices do not indicate the direction of firing rate changes. Therefore we compared error trial pre- and post-LP firing rates by dividing each cell's mean post-LP firing rate by its mean pre-LP rate.

\section{ENSEMBLE ACTIVITY STATE ANALYSIS}

To obtain an estimate of the neural firing rate for each isolated cell $i$ as a function of time bin $t$, $r_{i}(t)$, all spike trains were convolved with Gaussian kernels ( $\mathrm{SD}=500 / 4 \mathrm{~ms}$ ) and binned at $500 \mathrm{~ms}$ (approximately the inverse of the average firing rate of $\approx 2.4 \mathrm{~Hz}$ ). For population analysis,

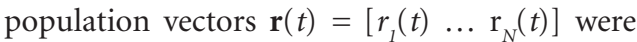
formed, with $N$ the number of single-units isolated from a given recording session and were plotted in a multi-dimensional space we called a 
multiple single-unit analysis (MSUA) space. The MSUA space refers to the $N$-dimensional space spanned by all recorded units and populated by these vectors $r(t)$. To obtain 3D projections of these $N$-dimensional MSUA spaces, we used multi-dimensional scaling for visualization (Figure 2A) as done previously (Lapish et al., 2008). Within these spaces, each point represents the entire state of the recorded network within one $500 \mathrm{~ms}$ bin, and all population vectors (points) corresponding to different $500 \mathrm{~ms}$ bins of the same behavioral period are shown in the same color.

To quantify behavioral effects on network activity, we computed the Mahalanobis distances (e.g., Krzanowski, 2000) between the sets of $N$-dimensional vectors associated with the different behavioral epochs, with covariance matrices pooled for the two conditions compared. Since all sessions were recorded from well trained animals and only a small number of error trials occurred, it was possible that the number of dimensions (neurons) approached the number of data points (time bins), and thus a regularized version of the covariance matrix was used as described in Hastie et al. (2009) to avoid singularity and statisticalreliability problems. As a simple means to rule out dimensionality or sample size differences from confounding comparisons between sessions, we restricted this analysis to only the four sessions with recording populations of greater than nine neurons. Also, for each set of samples to be compared we simply selected the same number of units and vector points. We limited the number of units for all analyses to the smallest ensemble size $\left(N_{\min }=5\right)$ for ensembles excluding "never theta" cells, since we were interested in comparing separation between these ensembles and those consisting of all recorded neurons. With $K_{\min }$ being the minimum number of data points across all samples $\left(K_{\min }=20\right.$; five trials $)$, as defined by the minimum number of error trials, $N_{\min }$ units and $K_{\min }$ data points were selected completely at random from the larger data sets and samples, and the Mahalanobis distances were computed. This procedure was repeated a 1000 times and results averaged to make full use of all units and data points recorded. Thus each comparison was performed on ensembles of the same size and over the same number of times bins. Both $t$-tests and non-parametric Wilcoxon rank sum tests were used to compare Mahalanobis distances between ensemble types.

\section{HIPPOCAMPAL THETA RESET}

The previous analysis (Hyman et al., 2010) did not find any differences in HPC theta power between correct and error periods, however this analysis looked at the entire trial period. Here we confined our analysis to theta activity only in the period surrounding each LP. For this analysis, local field potentials (LFP) for a period $2 \mathrm{~s}$ before and $2 \mathrm{~s}$ after each test LP were normalized by dividing by the session mean theta power. Next, normalized values of both correct and error trials, independently, were averaged for each $10 \mathrm{~ms}$ time bin. If a phase reset was present, theta oscillations should be visually identifiable in the averaged LFP. To statistically confirm this observation we computed power spectral densities (PSD) for the periods $0.8-1.8 \mathrm{~s}$ before the LP and $0.2-1.2 \mathrm{~s}$ after the LP (excluding the actual LP). A paired $t$-test compared values from the two PSDs in the theta range (7-11 Hz; peak frequency from the full error period $=8.2 \mathrm{~Hz}$ ). The mean normalized signal was also used to create spectrograms (2 ms time shifts; 256 frequency values; $20 \mathrm{~Hz}$ maximum frequency). Lastly, we filtered the LFP signals to remove non-theta band activity $(3-10 \mathrm{~Hz})$ and we computed the instantaneous phase of the LFP from each trial at $500 \mathrm{~ms}$ after the LP. We then examined the uniformity of these values with Rayleigh's test for both correct and error trials.

\section{RESULTS THETA-ENTRAINED CELLS ARE MORE TASK SELECTIVE THAN NEVER THETA-ENTRAINED mPFC CELLS}

Theta cells (27/74 total cells) were significantly more selective for both task general [ sample vs. test LP's; $t(156)=2.45 ; p<0.01 ; U=4772 ; p<0.04]$ and trial specific [left vs. right LP's; $t(156)=2.77$; $p<0.006 ; U=1279 ; p<0.002$ ] events than "never theta" cells (25/74 total cells; Figure 1A). This suggested that theta cells were more closely involved in correct DNMS task performance. A different picture emerged following error commission however. In this period "never theta" cells better differentiated erroneous test phase LP's than did theta-entrained cells [Figure 1A; $t(72)=2.14$; $p<0.035 ; U=773 ; p<0.007]$, even though the two groups were similarly selective for sample LP's on the same trials $[t(72)=0.5 ; p>0.62 ; U=1053$; $p>0.58]$. This finding suggested that "never theta" cells may have a unique post-error response role in the DNMS task. We next explored what exactly these cells were encoding.

\section{NEVER THETA CELLS HAVE SIGNIFICANT ERROR- EVOKED RESPONSES}

We compared pre- and post-test LP selectivity and found no main effects for cell type [theta or "never theta"; $F(107)=0.3 ; p>0.4$ ] or trial outcome [correct or error; $F(107)=0.7 ; p>0.58$ ], but the interaction of these two factors was significant 

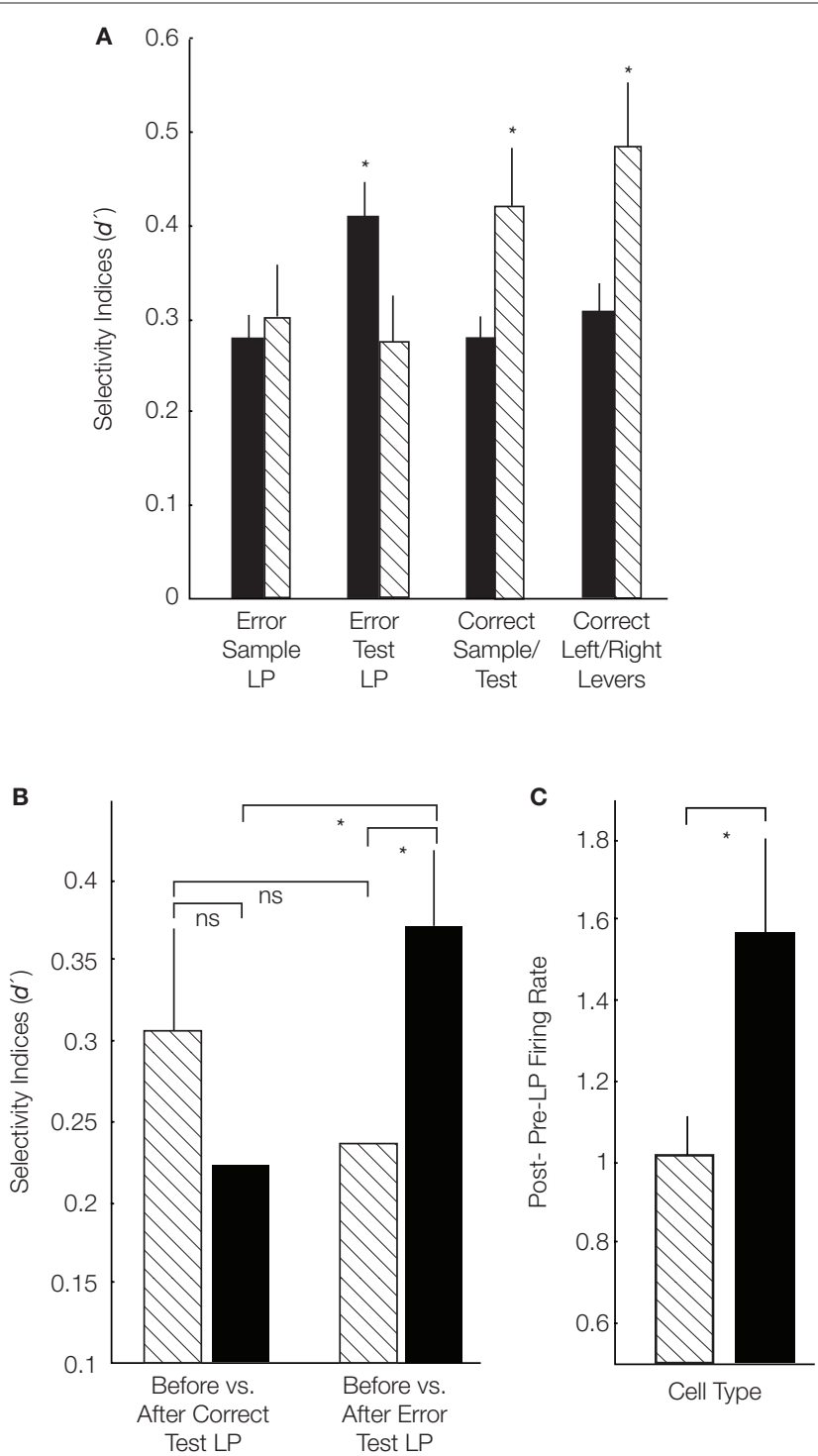

Cell Type

FIGURE 1 | Information encoding and post-error activity of mPFC units. In all plots black bars are for "never theta" cells and striped bars are theta cells. (A) Behavioral selectivity indices for "never theta" and theta cells. "Never theta" cells were significantly more selective for erroneous test LP's over inter-trial intervals, and theta cells were more selective for correct trial sample and test phases and left and right LPS $\left({ }^{*} p<0.05\right.$ $t$-test and Wilcoxon rank sum for grouped animal means; y-axis: group mean $d^{\prime}$ values and error bars: SEM). (B) Pre- vs. post-test LP selectivity by cell and trial type. There was a significant interaction between cell and trial types $(p<0.05$; two-way ANOVA). Both theta and "never theta" cells were equally selective for the periods before and after correct test LP's, while for error trials "never theta"

$[F(107)=5.3 ; p<0.02]$. Follow up paired $t$-tests and Wilcoxon rank sum tests revealed that "never theta" cells distinguished error trial pre- and post-LP periods more than during correct trials $[t(30)=3.16 ; p<0.004 ; U=792 ; p<0.009$; cells more strongly differentiated these periods ${ }^{*} p<0.05$ $t$-test and Wilcoxon rank sum for grouped animal means; $y$-axis: group mean $d^{\prime \prime}$ values and error bars: SEM). Furthermore, while theta cells were similarly selective for these periods on correct and error trials (ns; $p>0.05$ ), "never theta" cells selectivity significantly differed by trial type $\left({ }^{*} p<0.01 t\right.$-test and Wilcoxon rank sum for grouped animal means). (C) Error trial firing rates before and after test LP's. The $y$-axis shows the average of each cell's mean post-LP response divided by pre-LP firing rates, and accordingly values near 1.0 indicate similar firing rates before and after error LP's. "Never theta" firing rates increased (mean $=1.57 \pm 0.24$ ) but theta cell activity was stable (mean $=1.01 \pm 0.09 ;{ }^{*} p<0.05 t$-test and Wilcoxon rank sum for grouped animal means; error bars: SEM).

Figure 1B]. In contrast, theta-entrained cells were similarly selective irrespective of trial outcome $[t(22)=0.93 ; p>0.36 ; U=547 ; p>0.89$; Figure 1B]. Furthermore, on error trials "never theta" cells had higher degrees of selectivity 
between pre- and post-LP epochs than thetaentrained cells $[t(52)=2.1 ; p<0.03 ; U=518$; $p<0.042]$. No comparable differences were found for correct trials $[t(52)=1.19 ; p>0.24 ; U=675$; $p>0.46]$.

The results presented above show that "never theta" cells were more strongly affected by errors, but did not indicate whether activity increased or decreased following error commission. An analysis of pre- and post-error LP firing rates found that "never theta" cell rates increased (mean $\sim 1.5$ times greater than pre-LP) in the post-LP period and this increase was significantly greater than for theta-entrained cells $[t(52)=2.16 ; p<0.037$; $U=378 ; p<0.04$; Figure 1C]. The substantial increase in activity immediately after errors by never theta cells suggested that these cells are involved in error detection. As in the human and primate, error signals in the $\mathrm{mPFC}$ may alert other brain regions that an error has been made (Botvinick, 2007).

\section{NEVER THETA CELLS PROVIDE ERROR ENCODING FOR THE ENTIRE ENSEMBLE}

We next investigated how strongly "never theta" cells influenced the ensemble representation of the entire recorded mPFC population. Even though "never theta" cells that were analyzed above made up only $\sim 1 / 3$ of all neurons, they clearly modified ensemble states in MSUA space leading to the formation of distinguishable clusters of error test LP's (Figure 2A). In this plot dots represents the state of the entire recorded ensemble for each $500 \mathrm{~ms}$ bin, and bins corresponding to different behavioral period types are signified by different dot colors. Separation between sample and test responses is clearly visible but error sample responses are not distinct from correct samples. On the other hand, error test responses form a cluster in a distinct region of the test response area of the MSUA space.

Just how specific the role "never theta" cells play in the DNMS task was revealed when we compared separation distances (in the full multi-dimensional space, controlling for population size - see Materials and Methods) of the original ensembles with ensembles created by removing the "never theta" cells. In general, separation distances were not different between these two populations; more specifically comparisons of correct trial sample $[t(9)=2.01 ; p>0.07]$ and test LP's $[t(9)=1.86 ; p>0.1]$ and error trial sample LP's $[t(9)=1.37 ; p>0.2]$ were not significantly different (Figure 2B). Yet on the other hand, when never theta cells were excluded, the separation distances between error and correct test LP's was significantly decreased $[t(9)=2.5$; $p<0.03 ; U=18 ; p<0.03$ ], indicating that "never theta" cells played a highly significant role in the formation of ensemble representations of error responses.

\section{HPC THETA PHASE RESET}

We next switched from analyzing differences in unit firing rates, to looking for changes in field rhythms around errors. Specifically, we examined HPC theta activity before and after error test LP's for evidence of a phase reset given that theta resets have been observed in EEG recordings from humans after errors (Başar, 1980; Klimesch et al., 2004). LFP's from all error trials were normalized and averaged. We found that the 1-s period just after the LP had significantly greater mean power in the theta range than the 1-s period just before the $\operatorname{LP}[t(10)=3.03 ; p<0.006$; Figure $3 \mathrm{~A}]$. As can be seen in Figure 3B, the bulk of the increase in theta power occurred between $\sim 400$ and $600 \mathrm{~ms}$ following the LP.

The increase in theta power could be the result of a theta reset, yet the analysis above cannot prove this because spectral power is not phase dependent. However, if theta reset occurred, than in the averaged LFPs from many error trials (71 different error trials during nine recording sessions from four animals) out of phase theta activity should cancel, leaving only phase-locked theta activity. Indeed this was the case as the averaged LFP showed a clearly visible theta oscillation developing $\sim 400 \mathrm{~ms}$ after the LP that persists for three full theta cycles (Figure 3C). This indicated that theta activity was reset in a manner that was time-locked to the erroneous LP. Therefore even though behaviors were surely variable across this many different trials and animals, the theta reset was still clearly visible. The fact that instantaneous phases of LFP's from each trial at $535 \mathrm{~ms}$ after the LP (approximate time the largest peak appeared in the averaged LFP) were not uniformly distributed corroborated the visual observation of theta reset $\left(Z=24.25 ; p<5.4 \times 10^{-6}\right.$; Figure 3D). In marked contrast, no evidence of a theta reset was present in the averaged LFP surrounding correct LP on correct trials. However there was an apparent period of high-powered theta activity 1.5-2 s before the LP in the spectrogram (Figure 3E). This time period corresponds to the approximate time that the animal's path split between right and left lever trials, suggesting this might be the decision point. Therefore, in support of the findings of Jones and Wilson (2005a), theta power may increase at relevant periods of the task such as choice points. Nevertheless theta reset was observed only following errors on our task as there was no evidence of theta reset following correct 

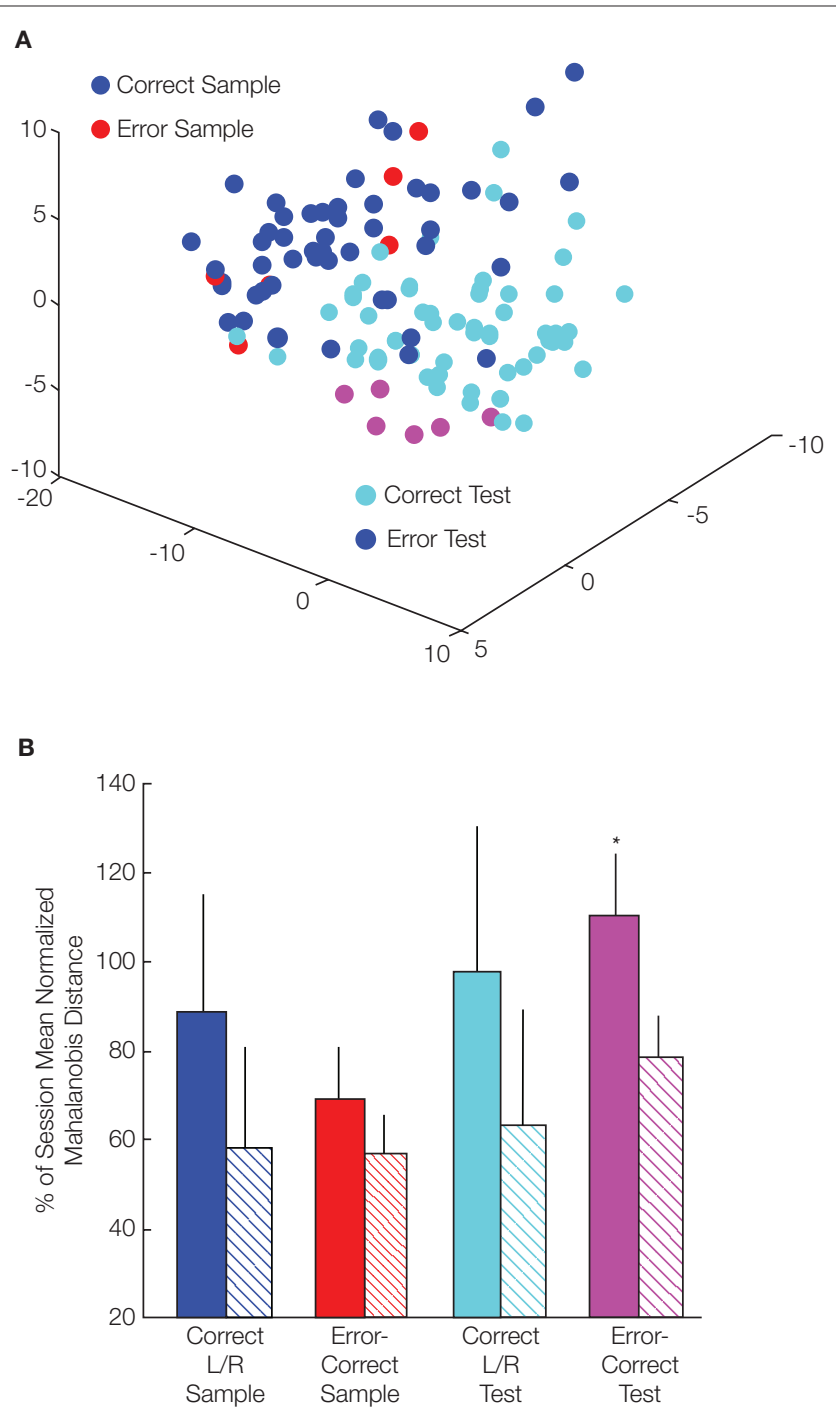

FIGURE 2 | Ensemble MSUA separation. (A) Example of 3D representation of MSUA space for the DNMS task. Population vectors are colored corresponding to the different task phase LP's and trial outcomes. The axes of this $3 \mathrm{D}$ projection correspond to different combinations of the single-unit firing rates. This plot shows clear clustering and separation of sample and test phase LP's on both error and correct trials, however only error trial test LP's separate from correct trials. (B) Mean MSUA separation distances for ensembles with (solid bars) and without "never theta" cells (striped bars). Full population ensembles had significantly greater separation of test LP's between error and correct trials than ensembles excluding "never theta" cells ${ }^{*} p<0.05$; $t$-test and Wilcoxon rank sum test for grouped animal means; $y$-axis: percentage of session mean Mahalanobis distance; error bars: SEM). response in the averaged LFP (Figure 3F) and the distribution of instantaneous phases $535 \mathrm{~ms}$ after correct trial LP's was uniform $(Z=1.39$; $p>0.49$; Figure 3G).

\section{LITERATURE REVIEW AND DISCUSSION INFORMATION ENCODING BY mPFC AND HPC NEURONS DURING WORKING MEMORY TASKS}

Aside from the encoding of purely spatial information by some HPC neurons, cells in the MPFC and HPC often respond to similar sensory, behavioral, and cognitive events. For example, when the same type of operant delayed response task is employed, both HPC and mPFC neurons respond in similar ways having correlates to $\mathrm{LP}$, right vs. left $\mathrm{LP}$, to nose pokes, to rewards, to task phases (sample vs. test), and so on (Hampson et al., 1993, Hampson and Deadwyler, 1996; Hyman et al., 2010). It therefore appears that both mPFC and HPC neurons can represent both trial specific and task universal elements of working memory tasks. However in these past studies there were no differences in the sample phase responses between correct and error trials. Thus, even though neurons in both the 


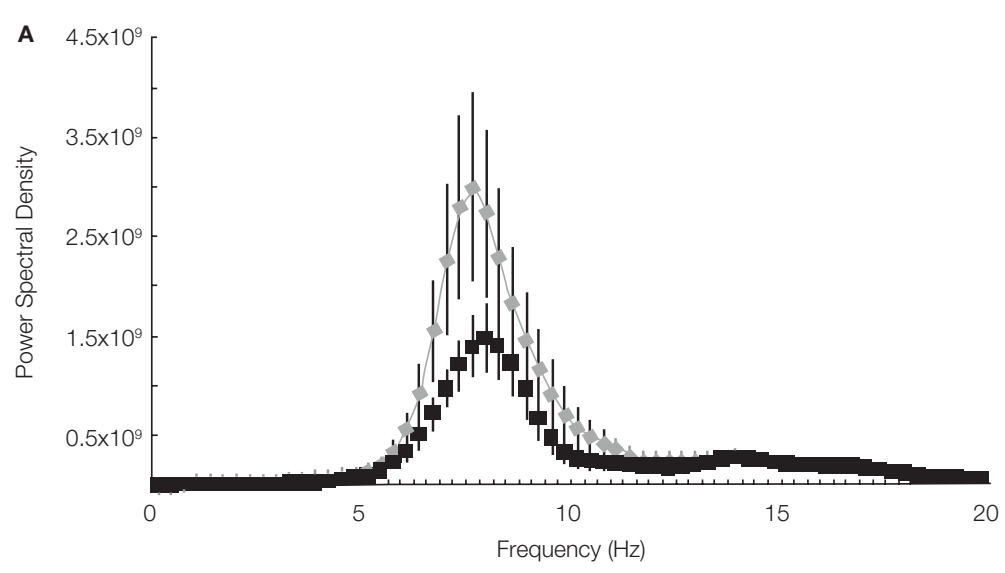

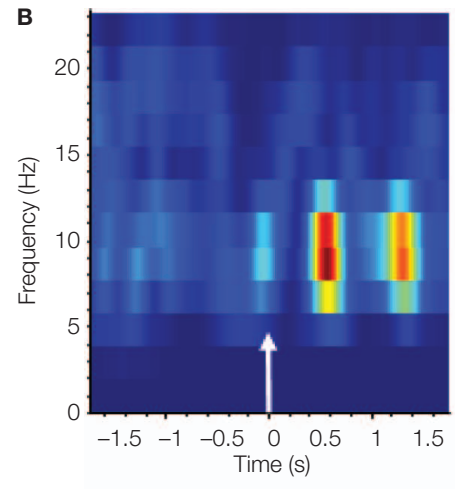

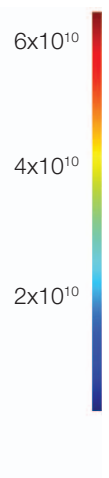

E
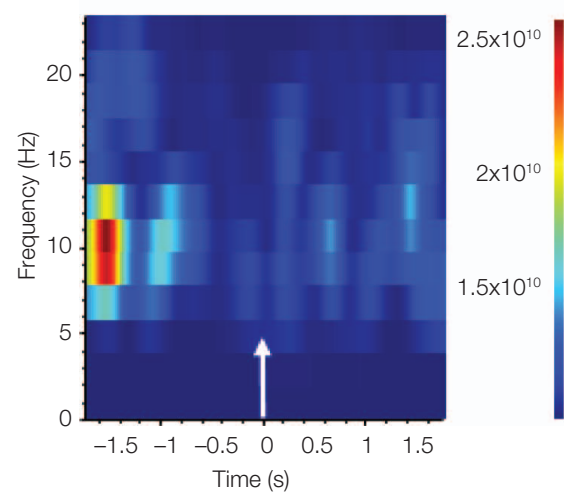

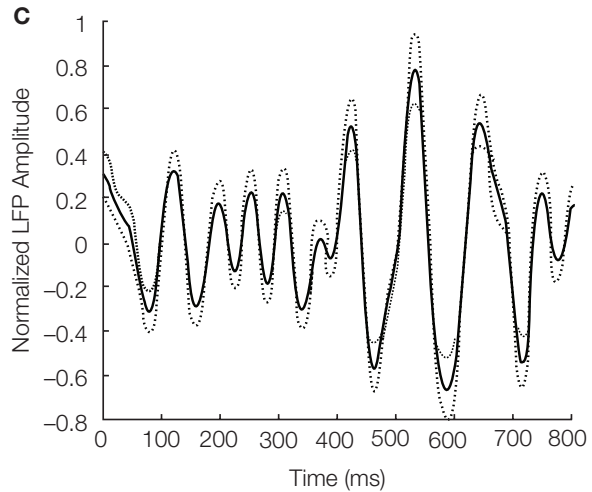

$\mathbf{F}$

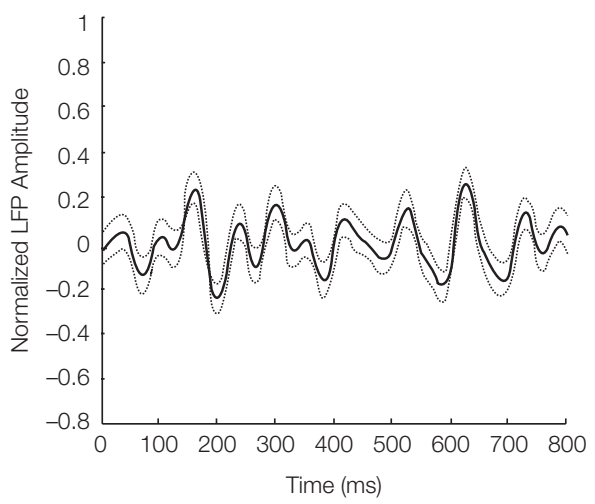

D

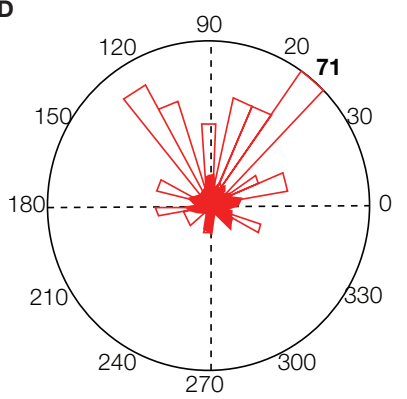

G

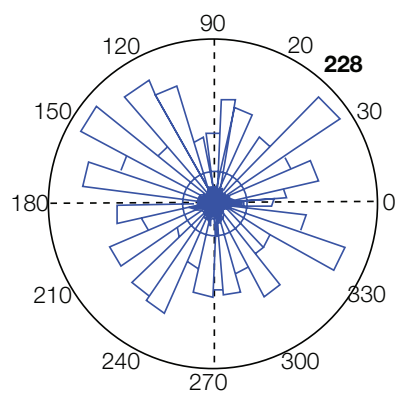

FIGURE 3 | Hippocampal theta phase reset after error responses. (A) Power spectral density distributions for $1 \mathrm{~s}$ before (black) and $1 \mathrm{~s}$ after (gray) erroneous test LP's (error bars: 95\% confidence intervals). The mean LFP theta power was significantly greater after the response ( $p<0.01$; paired $t$-test). (B) Spectrogram of erroneous test LP averaged LFPs. A clear increase in theta frequency power appears $\sim 400 \mathrm{~ms}$ following the response (shown by the arrow-timepoint $=0$ ). (C) Averaged normalized LFP signal for all error trials. Plot begins at the time of the LP. Averaged LFP (solid line) and \pm SEM (dotted lines) are shown. At $\sim 400 \mathrm{~ms}$ after the LP an obviously visible theta oscillation arises which is indicative aligned theta phases across the LFP's from each error trial and signifies that a theta phase reset occurred around the time of the LP. (D) Instantaneous theta phases of LFP's at 535 ms after error trial. Theta phases were not uniformly distributed ( $p<0.01$; Rayleigh's test of uniformity; bold number indicates the number of samples). (E) Spectrogram of correct trial test LP averaged LFP. There is a period of high theta power between 1.5 and $2 \mathrm{~s}$ before the LP (approximately the time locomotor trajectories split between right and left levers), indicating theta phase alignment at the decision point on correct trials. (F) Correct trial averaged normalized LFP. There are no signs of significant theta reset following correct LP's. (G) Instantaneous theta phases of LFP's at 535 ms after correct trial. Phases were distributed uniformly (Rayleigh's test of uniformity).
$\mathrm{mPFC}$ and HPC display responses that indicate that the sample LP was made and on which lever, these responses were not predictive of the eventual outcome of a trial. As will be discussed in greater detail below, the reason error-evoked responses were not found in Hyman et al. (2010) was because 
only cells phase-locked to HPC theta rhythms were analyzed in detail and theta-entrainment appears only in association with correct responses.

\section{THE mPFC AND HPC THETA RHYTHMS}

Anatomical studies have revealed a direct monosynaptic connection from the HPC (ventral CA1/subiculum) to the mPFC, along with dense reciprocal connections through the mediodorsal thalamus (Laroche et al., 1990; Vertes, 2006). The importance of this pathway was not entirely clear until Siapas et al. (2005) recorded neurons in the mPFC that were entrained to the theta rhythm oscillations (3-12 Hz) in HPC LFP during a variety of spatial tasks both with and without working memory components. This finding showed that a synchronous linkage is possible between these two areas, but the function was not readily apparent. Subsequent studies showed that HPC theta-entrainment of mPFC neurons: (1) is sensitive to sensory, behavioral, and environmental changes (Hyman et al., 2005), (2) is strongest at the decision point in a spatial working memory task (Jones and Wilson, 2005a), (3) creates a theta phase precession-like effect similar to HPC neurons (Jones and Wilson, 2005b), (4) can entrain mPFC gamma oscillations (Sirota et al., 2008), (5) is heightened during anxiety-related behaviors (Adhikari et al., 2010), (6) increases during the course of learning (Benchenane et al., 2010), and (7) is predictive of trial outcome during a working memory task (Hyman et al., 2010). From this literature it is evident that the entrainment of mPFC units to HPC theta oscillations provides a putative mechanism for a functional interaction between these regions.

Hyman et al. (2005) showed that the same mPFC neurons dynamically switch between firing states with and without HPC theta-entrainment. On a linear track, units were entrained during runs in one direction but not on return trips, and similarly neurons were entrained while foraging in one environment but not in another. In both instances there were groups of neurons entrained for each directional run on the linear track or foraging period in an environment, and a separate group of neurons entrained for runs in the opposite direction or other environment. This suggested that mPFC ensembles were affected by some combination of sensory, behavioral, and environmental encoding as well as HPC theta activity. Moreover, it implies that theta-entrainment may provide another method for representing information from simply firing rate changes.

Jones and Wilson (2005a) helped to clarify the role of theta-entrainment in coding information by investigating activity during a spatial working memory task. They recorded mPFC units and LFPs and HPC LFPs while animals ran an alternation task on a T-maze and found high levels of theta rhythm coherence and mPFC unit theta-entrainment as the animal approached the choice point. This suggested that the two areas were working together as the animal was making the correct choice between two responses in order to receive reinforcement. A second study from this group (Jones and Wilson, 2005b) showed that mPFC units exhibit phase precession-like effects as the animal ran down the stem of a T-maze. Spikes from these cells moved to progressively earlier phases of the HPC theta wave, similar to HPC units as animals walk through a place field (O'Keefe and Recce, 1993). Phase precession indicates that specific information is conveyed through the timing of action potentials with respect to the phase of HPC theta waves (Jensen and Lisman, 1996). Together these two studies reveal that $\mathrm{mPFC}$ units encode information via the temporal relationship between their spike trains and HPC theta oscillations.

Even though theta-entrainment increases on correct trials (Jones and Wilson, 2005a), it should be emphasized that this does not imply it is a means of sending specific information from the HPC to the mPFC. In Hyman et al. (2010) we showed that theta-entrained cells were firing in a similar manner to task events on correct and error trials even though they lost their thetaentrainment on error trials. Therefore, the mPFC appeared to possess the requisite information required to make a correct response, but the lack of theta-entrainment for some reason prevented the animal from doing so. We were interested in why this might be the case and so performed a re-analysis of the Hyman et al. (2010) data set to gain insights into the differences in the processing states associated with correct vs. incorrect trials.

\section{RE-ANALYSIS OF HYMAN ET AL. (2010)}

The current re-analysis revealed that theta cells seem to be carrying the bulk of the information necessary for successful DNMS performance (Figure 1A). We had previously found that the firing rates of these same cells did not differentiate error and correct trials for either sample or test LP's, yet they lost theta-entrainment on error trials (Hyman et al., 2010). Together these findings suggest that the robust behaviorally correlated firing patterns in MPFC neurons are not merely the product of increased HPC input during periods of entrainment. Rather thetaentrainment likely represents the coordination of HPC and mPFC activity necessary for correct task performance (See Key Concepts). In this 
light, transient periods of theta-entrainment may aid in decision making on correct trials, as implied by the phase synchronized HPC theta oscillations during the approach to the lever panel on the test phase (Figure 3E). Also, previous results have shown increased $\mathrm{mPFC}$ unit theta-entrainment, LFP theta coherence and increased correlations between $\mathrm{MPFC}$ and HPC cell pairs at the choice point of T-maze working memory task on correct trials (Jones and Wilson, 2005a). Furthermore, as an animal learns, theta coherence, phase-locking, mPFC cell pair crosscorrelations, and $\mathrm{mPFC}$ cell assembly replay during HPC sharp waves all increase (Benchenane et al., 2010). Notably, the theta-entrained mPFC cells have the strongest encoding of task-relevant information (Figure 1A) and carry more information during periods of strong entrainment (Jones and Wilson, 2005a). These findings illustrate the importance of mPFC-HPC theta coherence and entrainment during working memory tasks for the coordination of activity between the two areas (See Key Concepts).

It is easy to envision the role of $\mathrm{mPFC}-\mathrm{HPC}$ theta interactions during learning for forming long-term memories by acting as tag for rewardrelated activity (Benchenane et al., 2010). But what is the importance on a trial-by-trial basis during steady state performance of a well-learned task, as is the case for many working memory tasks? Although we discussed evidence above that the mPFC and HPC encode similar aspects of working memory tasks, their overall perspective on the situation is likely different. The HPC contains place neurons that become active when the animal is in a particular region of an environment (O'Keefe and Dostrovsky, 1971). These place cells collectively tile an environment and provide a spatial map informing the animal of its location relative to the overall spatial layout (Wilson and McNaughton, 1993; Eichenbaum, 2004). Recently we have explored how mPFC neurons encode spatial environments and found that individual neurons had poor spatial selectivity, in accord with previous studies (Poucet, 1998; Gemmell et al., 2002; Hok et al., 2005). On the other hand, large ensembles of $\mathrm{mPFC}$ neurons formed highly distributed representations of entire environments that were independent of the rat's specific location in the environments (Seamans et al., 2009). Furthermore, the representation changed based on personally relevant factors such as familiarity, cues, objects placed in the environments and rewards. These data suggest that while HPC networks provide information about one's specific location within an environment, $\mathrm{mPFC}$ networks provide a more holistic and egocentric represen- tation of the entire environment and what is personally relevant based on current actions, goals and past experience. In order to respond appropriately, the egocentric representation provided by the MPFC might need to be aligned with the more allocentric representation provided by the HPC and perhaps during steady state performance one function of theta-entrainment is to align these two representations.

\section{ERROR PROCESSING BY mPFC NEURONS AND ENSEMBLES}

In the present study we also examined the "never theta" segment of the population that was neglected in Hyman et al. (2010). Previously we had found that individual theta cells did not differentiate between correct and error trials. In the current analysis we used population-level analyses and found that ensembles containing only theta cells differentiated correct trial phases and lever locations just as well as the full ensembles (Figures 2A,B). It was only test phase error and test phase correct responses that were more distinguishable by ensembles that included both theta and "never theta" cells. It therefore appeared that the primary role of "never theta" cells in the DNMS task was to encode the commission of errors (See Key Concepts). The presence of error encoding neurons in the mPFC is not new. For instance, Narayanan and Laubach (2008) found error-related correlates in the $\mathrm{mPFC}$ using a reaction time task. It is also of note that we found virtually the same percentage of error-related neurons as these authors using a completely different task. Narayanan and Laubach (2008) also showed that error-related responses persisted over the inter-trial interval and so they postulated that this activity might represent a form of retrospective working memory for trial outcomes. Error-related activity has also been recorded from medial frontal neurons of primates (Ito et al., 2003). While error-related activity in the $\mathrm{mPFC}$ is not new, what was novel here was that the specific set of error neurons also happened to be those that were never theta entrained.

\section{ERROR-EVOKED HIPPOCAMPAL THETA RESET}

The emergence of activity of mPFC "error" neurons was not the only significant event that emerged after an error was made. Additionally we found that there was also a reset of the HPC theta rhythm at the same time (Figure 3). HPC theta resets have been previously found following stimulus presentations or electrical stimulation of various afferent pathways (Brazhnik et al., 1985; Vinogradova et al., 1993; Givens, 1996; Tesche and Karhu, 2000; Williams and Givens, 2003). 
Furthermore, there is a long history of scalp EEG recordings documenting phase resets in the theta range following error commission (Başar, 1980; Klimesch et al., 2004). In our re-analysis of Hyman et al. (2010) we found clear evidence of an increase in HPC theta power following error responses (Figures 3A-C) (See Key Concepts). The unmistakable signature of a theta phase reset, pronounced theta oscillations in the averaged LFP, appeared $\sim 400 \mathrm{~ms}$ after the error test LP, consistent with the time lag found in previous reports (Givens, 1996; Williams and Givens, 2003). In our case, this reset occurred within the HPC, which is significant because in rats the vast majority of the theta signal in mPFC is generated by the HPC (Sirota et al., 2008). Therefore, the present study provides some support for the theory that an error-associated response, such as the errorrelated negativity, could be generated by a theta reset (Başar, 1980; Klimesch et al., 2004).

The functional significance of theta resets in this context is not immediately obvious. Theta resets are believed to be a mechanism for phase-locking HPC activity to behaviorally relevant events and thereby may enhance cognitive processing (Givens, 1996; Hasselmo, 2007, 2008). It has also been found that theta resets create the optimal conditions for long-term potentiation (McCartney et al., 2004), so that cellular responses occurring subsequent to behavioral or sensory events will undergo lasting synaptic alterations. In the present study, after an error response there was no immediately impending sensory stimuli or necessary behaviors to encode, leaving only the error itself as the relevant event. A theta reset at this point may ensure the subsequent lack of reinforcement would be strongly encoded. In addition, as argued above, if a loss of thetaentrainment dissociated the ego- and allocentric spatial representations provided by the $\mathrm{MPFC}$ and HPC respectively, a theta reset after an error may serve as a means of reintegration of these two representations.

\section{RELATING HUMAN mPFC THETA ACTIVITY TO RODENT STUDIES}

In humans, EEG or MEG measurements have revealed a strong theta signal over the midline frontal cortex (Sasaki et al., 1996; Gevins et al., 1997; Asada et al., 1999; Ishii et al., 1999; Kahana et al., 1999; Jensen and Tesche, 2002; Onton et al., 2005; Delorme et al., 2007). In most human studies this "frontal midline theta" $(\mathrm{fm} \theta)$ has a peak in the $5-7 \mathrm{~Hz}$ range. This is a slightly lower frequency than typically found in walking/running rodents $(7-10 \mathrm{~Hz})$ though lower frequencies $(4-7 \mathrm{~Hz})$ have been found in the absence of locomotion such as during freezing or orienting (Kramis et al., 1975). Human $\mathrm{fm} \theta$ is stronger on average during waking, various types of demanding cognitive tasks such as mental calculation, concentration, movement preparation, short-term memory, or with heightened or sustained attention, and error commission (Sasaki et al., 1996; Gevins et al., 1997; Ishii et al., 1999; Kahana et al., 1999; Jensen and Tesche, 2002; Delorme et al., 2007; Marco-Pallarés et al., 2008; Cavanaugh et al., 2010). Its strength increases in relation to memory load (Gevins et al., 1997; Michels et al., 2008) and with greater cognitive demand, such as during incongruent trials of the Stroop task or with switch trials on set-shifting tasks (Sauseng et al., 2006; Hanslmayr et al., 2008).

The site of origin of fm $\theta$ in humans appears to be in or near the dorsal anterior cingulate cortex (ACC; Gevins et al., 1997; Asada et al., 1999; Ishii et al., 1999; Onton et al., 2005). In fact the fm $\theta$ contributed close to half of the total theta power measured from $\mathrm{Fz}$, while anterior and posterior regions contributed only $\sim 20 \%$ of total theta power (Onton et al., 2005). The human ACC where $\operatorname{fm} \theta$ is centered includes Brodmann's areas 24, 32, and 25 (Vogt and Vogt, 2003), which is anatomically equivalent to the anterior cingulate, prelimbic, and infralimbic cortices respectively in rats. Therefore, the anatomical location where $\mathrm{fm} \theta$ is centered in humans is anatomically related to the regions of the rat $\mathrm{mPFC}$ recorded in the present study.

One of the most significant differences between the human and rat in terms of frontal theta is the relative contribution of the HPC. Unlike the rodent, in humans the HPC theta signal, while present, is relatively weak and HPC and cortical theta are not reliably synchronized at rest (Kahana et al., 2001; Cantero et al., 2003; Ekstrom et al., 2005). However the hippocampus does provide input to the midline frontal cortices and theta in the two regions can become synchronized under certain conditions, including during working memory tasks or with task switching (Miller, 1991; Sarnthein et al., 1998; von Stein and Sarnthein, 2000; Raghavachari et al., 2001; Sarnthein et al., 2006; Sauseng et al., 2006; Anderson et al., 2010). Although the ACC may be a main driver of $\operatorname{fm} \theta$ in humans, a recent study employing multi-site recordings and Granger causality provided evidence of directionality, with medial temporal lobe theta potentially driving $\operatorname{fm} \theta$ in humans (Anderson et al., 2010).

There is an interesting relationship between $\mathrm{fm} \theta$ and neural activity in the mPFC. It is often the case that theta increases in medial frontal cortex are correlated negatively with the BOLD signal in 
that region (Mizuhara et al., 2004; Meltzer et al., 2007; Mizuhara and Yamaguchi, 2007). This may not be too surprising given that this region is considered part of the "default mode network" where increases in memory and attention demands often elicit negative BOLD signal changes (Raichle et al., 2001). As further support for this type of inverse relationship, Wang et al. (2005) observed that theta increases in the ACC during a variety of cognitive tasks were accompanied by a decrease in multi-unit spiking of superficial cortical neurons. The converse is also true as theta power in the human $\mathrm{mPFC}$ was found to be diminished on trials preceding an error (Cavanaugh et al., 2009), which is the same time interval other studies have shown there to be an increase in default mode network activity (Weissman et al., 2006; Eichele et al., 2008).

Activity in the default mode network activity may represent the shifting of attention away from the task at hand and toward internal processing streams (Buckner et al., 2008), while an increase in theta may be associated with enhanced attention for task elements, as reviewed above. Therefore, it makes sense that these two processes should be dissociated. However, there is one situation where they are positively correlated. Specifically after an error is committed, there is both an increase in the BOLD signal recorded from the ACC (Carter et al., 1998; van Veen and Carter, 2002) and also an increase in $\mathrm{fm} \theta$ (Cavanaugh et al., 2009). The period following an error is somewhat unique in that it is the time when the ERN is observed, which is also thought to be generated within the ACC (van Veen and Carter, 2002; See Key Concepts). Concurrent increases in $\operatorname{fm} \theta$ and ACC activity following errors may represent an active comparison between the results of one's own internal processing (default mode network activity) in relation to newly acquired information garnered from the redirection of attention back to the task $(f m \theta)$. In other words, errors are a special situation requiring an evaluation of the current task situation in relation to internal representations of expected outcomes.

\section{A THEORY OF TASK AND ERROR ENCODING BY HPC/ MPFC NETWORKS}

It is becoming increasingly evident that neurons in the mPFC exhibit correlates to almost everything the rat experiences. For instance Jung et al. (1998) observed over 70 correlates for mPFC neurons, including correlates for diverse actions and action sequences, cues rewards, and so on. As noted above, there are also strong $\mathrm{mPFC}$ correlates to errors (Narayanan and Laubach, 2008). On working memory tasks, mPFC neurons exhibit various forms of delay period and response related activity as well as activity correlated to slight variations in an animal's path or changes in body position (Euston and McNaughton, 2006; Narayanan and Laubach, 2006, 2009; Cowen and McNaughton, 2007). Furthermore, we have observed that mPFC ensembles can represent each separate sub-component of a working memory task, cues, and rules on a set-shifting task as well as entire environments through entering unique activity states (Lapish et al., 2008; Seamans et al., 2009; Durstewitz et al., 2010; Hyman et al., 2010). It would appear that the mPFC contains representations of almost all task-related experiences and actions, which is consistent with the proposed general role of the ACC in task and action monitoring (Botvinick, 2007).

One potentially interesting finding of the present study was that neurons with the strongest task correlates also tended to be those that were phase-locked to HPC theta. One possibility is that since the $\mathrm{mPFC}$ represents such a vast array of information, periods of theta-entrainment may serve as a means to draw attention to only those representations that are task-relevant. Thus, the loss of entrainment on error trials in Hyman et al. (2010) was not due to a loss of information about the task itself (i.e., task-related firing rates were unchanged) but rather because attention was no longer focused on these task-relevant representations. In contrast to theta-entrained neurons, "never theta" cells responded selectively when the animal had committed an error. These neurons appeared to be relatively unconcerned with the representation of the other task variables required to perform the task correctly. It could be that these neurons might encode more internalized variables, such as the realization that the rat's internalized model of the world was incorrect. In addition to activation of this pool of neurons around errors, we also observed a significant increase in theta power and a theta reset. Again, this reset might serve to refocus attention on task-relevant representations or to realign $\mathrm{HPC}$ and $\mathrm{mPFC}$ representations. The concurrent theta reset along with the activation of "never theta" neurons may collectively serve as a means to compare the rat's current internal representation of the world with newly acquired information garnered from the redirection of attention back to the task (i.e., the realization that an expected reward was not forthcoming). In this way, synchrony or entrainment to various rhythms may not be a means of information transfer but a way for the brain to select certain representations from the vast array of representations encoded by mPFC ensembles. 


\section{CONCLUSION}

The present re-analysis of Hyman et al. (2010) has shown that errors have a large impact of processing states in both the mPFC and HPC. Even though mPFC neurons are not reliant upon HPC theta frequency input to form firing rate-based representations of a working memory task, the absence of significant HPC theta synchrony greatly impairs performance. During correct trials mPFC-HPC theta interactions may create the appropriate dynamics for the integration or comparison of distinct representations provided by these two areas. Following error commission two distinct effects occurred: (1) the "never theta" cells, which are of minimal influence during correct trials, vault into prominence creating a unique post-error ensemble activity state; and concurrently (2) the HPC theta rhythm resets. Together these produce a multiple component reaction that could serve as an error signal in $\mathrm{MPFC}$, HPC, and beyond.

\section{REFERENCES}

Adhikari, A., Topiwala, M.A., and Gordon, J. A. (2010). Synchronized activity between the ventral hippocampus and the medial prefrontal cortex during anxiety. Neuron 65, 257-269.

Anderson, K. L., Rajagovindan, R., Ghacibeh, G. A., Meador, K. J., and Ding, M. (2010). Theta oscillations mediate interaction between prefrontal cortex and medial temporal lobe in human memory. Cereb. Cortex 20, 1604-1612.

Asada, H., Fukuda, Y., Tsunoda, S., Yamaguchi, M., and Tonoike, M. (1999). Frontal midline theta rhythms reflect alternative activation of prefrontal cortex and anterior cingulate cortex in humans. Neurosci. Lett. 274 29-32.

Başar, E. (1980). EEG Brain Dynamics: Relation Between EEG and BrainEvoked Potentials. Amsterdam: Elsevier.

Benchenane, K., Peyrache, A., Khamassi, M., Tierney, P. L., Gioanni, Y., Battaglia, F.P., and Wiener, S. I. (2010). Coherent theta oscillations and reorganization of spike timing in the hippocampalprefrontal network upon learning. Neuron 66, 921-936.

Botvinick,M.M. (2007).Conflict monitoring and decision making: Reconciling two perspectives on anterior cingulate function. Cogn. Affect. Behav. Neurosci. 7, 356-366.

Brazhnik, E. S., Vinogradova, O. S., and Karanov, A. M. (1985). Frequency modulation of neuronal thetabursts in rabbit's septum by lowfrequency repetitive stimulation of the afferent pathways. Neuroscience $14,501-508$.

Buckner, R. L., Andrews-Hanna, J. R., and Schacter, D. L. (2008). The brain's default network: Anatomy, function, and relevance to disease. Ann. N. Y. Acad. Sci. 1124, 1-38.

Cantero, J. L., Atienza, M., Stickgold, R., Kahana, M. J., Madsen, J. R., and Kocsis, B. (2003). Sleep-dependent theta oscillations in the human hippocampus and neocortex. J. Neurosci. 23, 10897-10903.

Carter, C. S., Braver, T. S., Barch, D. M., Botvinick, M.M., Noll, D., and Cohen,
J. D. (1998). Anterior cingulate cortex, error detection, and the online monitoring of performance. Science 280, 747-749.

Cavanaugh, J. F., Cohen, M. X., and Allen, J. J. B. (2009). Prelude to and resolution of an error: EEG phase synchrony reveals cognitive control dynamics during action monitoring. J. Neurosci. 29, 98-105.

Cavanaugh, J. F., Frank, M. J., Klein, T. J., and Allen, J. J. B. (2010). Frontal theta links prediction errors to behavioral adaptation in reinforcement learning. Neuroimage 49, 3198-3209.

Cowen, S. L., and McNaughton, B. L. (2007). Selective delay activity in the medial prefrontal cortex of the rat: Contribution of sensorimotor information and contingency. J. Neurophysiol. 98, 303-316.

Delorme, A., Westerfield, M., and Makeig, S. (2007). Medial prefrontal theta bursts precede rapid motor responses during visual selective attention. $J$. Neurosci. 27, 11949-11959.

Durstewitz, D., Vittoz, N. M., Floresco, S. B., and Seamans, J. K. (2010). Abrupt transitions between prefrontal neural ensemble states accompany behavioral transitions during rule learning. Neuron 66, 438-448.

Eichele, T., Debener, S., Calhoun, V. D., Specht, K., Engel, A. K., Hugdahi, K., von Cramon, D. Y., and Ullsperger, M. (2008). Prediction of human errors by maladaptive changes in event-related brain networks. Proc. Natl. Acad. Sci. U.S.A. 105, 6173-6178.

Eichenbaum, H. (2004). Hippocampus: cognitive processes and neural representations that underlie declarative memory. Neuron 44, 109-120.

Ekstrom, A. D., Caplan, J. B., Ho, E., Shattuck, K., Fried, I., and Kahana, M. J. (2005). Human hippocampal theta activity during virtual navigation. Hippocampus 15, 881-889.

Euston, D. R., and McNaughton, B. L. (2006). Apparent encoding of sequential context in rat medial prefrontal cortex is accounted for by behavioral variability. J. Neurosci. 26,13143-13155.
Gemmell, C., Anderson, M., and O'Mara, S. M. (2002). Deep layer prefrontal cortex unit discharge in a cuecontrolled open-field environment in the freely-moving rat. Behav. Brain Res. 133, 1-10.

Gevins, A., Smith, M. E., McEvoy, L., and Yu, D. (1997). High-resolution EEG mapping of cortical activation related to working memory: Effects of task difficulty, type of processing, and practice. Cereb. Cortex 7, 374-385.

Givens, B. (1996). Stimulus-evoked resetting of the dentate theta rhythm: Relation to working memory. Neuroreport 8, 159-163.

Hampson, R. E., and Deadwyler, S. A. (1996). Ensemble codes involving hippocampal neurons are at risk during delayed performance tests. Proc. Natl. Acad. Sci. U.S.A. 93, 13487-13493.

Hampson, R. E., Heyser, C. J., and Deadwyler, S.A. (1993). Hippocampal cell firing correlates of delayed-matchto-sample performance in the rat Behav. Neurosci. 107, 715-739.

Hanslmayr, S., Pastötter, B., Bäuml, K. Gruber, S., Wimber, M., and Klimesch, W. (2008). The electrophysiological dynamics of interference during the Stroop task. J. Cogn. Neurosci. 20 215-225.

Hasselmo, M. E. (2007). Arc length coding by interference of theta frequency oscillations may underlie contextdependent hippocampal unit data and episodic memory function. Learn. Mem. 14, 782-794.

Hasselmo, M. E. (2008). Grid cell mechanisms and function: Contributions of entorhinal persistent spiking and phase resetting. Hippocampus 18 1213-1229.

Hastie, T., Tibshirani, R., and Friedman, J. (2009). The Elements of Statistical Learning: Data Mining, Inference, and Prediction. New York: Springer Science.

Hok, V., Save, E., Lenck-Santini, P. P., and Poucet, B. (2005). Coding for spatial goals in the prelimbic/infralimbic area of the rat frontal cortex. Proc. Natl. Acad. Sci. U.S.A. 102, 4602-4607.

Hyman, J. M., Zilli, E. A., Paley, A. M., and Hasselmo, M. E. (2005). Medial prefrontal cortex cells show dynamic modulation with the hippocampal theta rhythm dependent on behavior. Hippocampus 15, 739-749.

Hyman, J. M., Zilli, E. A., Paley, A. M. and Hasselmo, M. E. (2010). Working memory performance correlates with prefrontal-hippocampal theta interactions but not with prefrontal neuron firing rates. Front. Integr. Neurosci. 4:2. doi: 10.3389/neuro.07.002.2010

Ishii, R., Shinosaki, K., Ukai, S., Inouye, T., Ishihara, T., Yoshimine, T., Hirabuki, N., Asada, H., Kihara, T., Robinson, S. E., and Takeda, M. (1999). Medial prefrontal cortex generates frontal midline theta rhythm. Neuroreport 10, 675-679.

Ito, S., Stuphorn, V., Brown, J. W., and Schall, J. D. (2003). Performance monitoring by the anterior cingulate cortex during saccade countermanding. Science 302, 120-122.

Jensen, O., and Lisman, J. E. (1996). Hippocampal CA3 region predicts memory sequences: Accounting for the phase precession of place cells. Learn. Mem. 3, 264-278.

Jensen, O., and Tesche, C. D. (2002). Frontal theta activity in humans increases with memory load in a working memory task. Eur. J. Neurosci. 15, 1395-1399.

Jones, M. W., and Wilson, M. A. (2005a). Theta rhythms coordinate hippocampal-prefrontal interactions in a spatial memory task. PLoS Biol. 3, e402. doi: 10.1371/journal.pbio.0030402

Jones, M. W., and Wilson, M. A. (2005b). Phase precession of medial prefrontal cortical activity relative to the hippocampal theta rhythm. Hippocampus 15 , 867-873.

Jung, M. W., Qin, Y., McNaughton, B. L., and Barnes, C. A. (1998). Firing characteristics of deep layer neurons in prefrontal cortex in rats performing spatial working memory tasks. Cereb. Cortex 8, 437-450.

Kahana, M. J., Seelig, D., and Madsen, J. R. (2001). Theta returns. Curr. Opin. Neurobiol. 11, 739-744.

Kahana, M. J., Sekuler, R., Caplan, J. B. Kirschen, M., and Madsen, J.R. (1999). Human theta oscillations exhibit task 
dependence during virtual maze navigation. Nature 399, 781-784.

Klimesch, W., Schack, B., Schabus, M., Doppelmayr, M., Gruber, W., and Sauseng, P. (2004). Phase-locked alpha and theta oscillations generate the p1-n 1 complex and are related to memory performance. Brain Res. Cogn. Brain Res. 19, 302-316.

Kramis, R., Vanderwolf, C. H., and Bland, B. H. (1975). Two types of hippocampal rhythmical slow activity in both the rabbit and the rat: Relation to behavior and effects of atropine, diethyl ether, urethane, and pentobarbital. Exp. Neurol. 49, 58-85.

Krzanowski, W. J. (2000). Principles of Multivariate Analysis: A User's Perspective. New York: Oxford University Press.

Lapish, C. C., Durstewitz, D., Chandler, L. J., and Seamans, J.K. (2008). Successful choice behavior is associated with distinct and coherent network states in anterior cingulate cortex. Proc. Natl. Acad. Sci. U.S.A. 105, 11963-11968.

Laroche, S., Davis, S., Jay, T. M., and Thierry, A. M. (1990). Long-term potentiation in the prefrontal cortex following stimulation of the hippocampal CA1/subicular region. Neurosci. Lett. 114, 184-190.

Marco-Pallarés, J., Camara, E., Münte, T. F., and Rodríguez-Fornells, A. (2008). Neural mechanisms underlying adaptive actions after slips. J. Cogn. Neurosci. 20, 1595-1610.

McCartney, H., Johnson, A. D., Weil, Z. M., and Givens, B. (2004). Theta reset produces optimal conditions for longterm potentiation. Hippocampus 14, 684-687.

Meltzer, J. A., Negishi, M., Mayes, L. C., and Constable, R. T. (2007). Individual differences in EEG theta and alpha dynamics during working memory correlate with fMRI responses across subjects. Clin. Neuropsychol. 118, 2419-2436.

Michels, L., Moazami-Goudarzi, M., Jeanmonod, D., and Sarnthein, J. (2008). EEG alpha distinguishes between cuneal and precuneal activation in working memory. Neuroimage 40, 1296-1310.

Miller, E. K., Freedman, D. J., and Wallis, J. D. (2002). The prefrontal cortex: categories, concepts and cognition.
Philos. Trans. R. Soc. Lond. B Biol. Sci. 357, 1123-1136.

Miller, R. (1991). Cortico-Hippocampal Interplay and the Representation of Contexts in the Brain. (Studies in brain function no. 17). Berlin: Springer.

Mizuhara, H., Wang, L. Q., Kobayashi, K., and Yamaguchi, Y. (2004). A longrange cortical network emerging with theta oscillation in a mental task. Neuroreport 15, 1233-1238.

Mizuhara, H., and Yamaguchi, Y. (2007). Human cortical circuits for central executive function emerge by theta phase synchronization. Neuroimage $36,232-244$.

Narayanan, N. S., and Laubach, M. (2006). Top-down control of motor cortex ensembles by dorsomedial prefrontal cortex. Neuron 7, 921-931.

Narayanan, N. S., and Laubach, M. (2008). Neuronal correlates of posterror slowing in the rat dorsomedial prefrontal cortex. J. Neurophysiol. 100, 520-525.

Narayanan, N. S., and Laubach, M. (2009). Delay activity in rodent frontal cortex during a simple reaction time task. J. Neurophysiol. 101, 2859-2871.

O'Keefe, J., and Dostrovsky, J. (1971). The hippocampus as a spatial map. Preliminary evidence from unit activity in the freely-moving rat. Brain Res. 34, 171-175.

O'Keefe, J., and Recce, M. L. (1993). Phase relationship between hippocampal place units and the EEG theta rhythm. Hippocampus 3, 317-330.

Onton, J., Delorme, A., and Makeig, S. (2005). Frontal midline EEG dynamics during working memory. Neuroimage 27, 341-356.

Poucet, B. (1998). Searching for spatial unit firing in the prelimbic area of the rat medial prefrontal cortex. Behav. Brain Res. 84, 151-159.

Raghavachari, S. Kahana, M. J., Rizzuto, D. S., Caplan, J. B., Kirschen, M. P., Bourgeois, B., Madsen, J. R., and Lisman, J. E. (2001). Gating of human theta oscillations by a working memory task. J. Neurosci. 21, 3175-3183.

Raichle, M.E., MacLeod, A. M., Snyder,A. Z., Powers, W. J., Gusnard, D. A., and Shulman, G.L. (2001). A default mode of brain function. Proc. Natl. Acad. Sci. U.S.A. $98,676-682$.
Sarnthein, J., Petsche, H., Rappelsberger, P., Shaw, G. L., and von Stein, A (1998). Synchronization between prefrontal and posterior association cortex during human working memory. Proc. Natl. Acad. Sci. U.S.A. 95, 7092-7096.

Sarnthein, J., Stern, J. Aufenberg, C., Rousson, V., and Jeanmonod, D. (2006). Increased EEG power and slowed dominant frequency in patients with neurogenic pain. Brain 129, 55-64.

Sasaki, K., Nambu, A., Tsujimoto, T. Matsuzaki, R., Kyuhou, S., and Gemba, H. (1996). Studies on integrative functions of the human frontal association cortex with MEG. Brain Res. Cogn. Brain Res. 5, 165-174.

Sauseng, P., Klimesch, W., Freunberger, R. Pecherstorfer, T., Hanslmayr, S., and Doppelmayr, M. (2006). Relevance of EEG alpha and theta oscillations during task switching. Exp. Brain Res 170, 295-301.

Seamans, J. K., Hyman, J. M., Lapish, C. C., Balaguer-Ballester, E., Ma, L., and Durstewitz, D. (2009). A prefrontal cortex network representation of mental set in rats. Abstr. Soc. Neurosci. 579.9.

Siapas, A. G., Lubenov, E. V., and Wilson, M. A. (2005). Prefrontal phase locking to hippocampal theta oscillations. Neuron 46, 141-151.

Sirota, A., Montgomery, S., Fujisawa, S. Isomura, Y., Zugaro, M., and Buzsaki, G. (2008). Entrainment of neocortical neurons and gamma oscillations by the hippocampal theta rhythm. Neuron 60, 683-697.

Tesche, C. D., and Karhu, J. (2000). Theta oscillations index human hippocampal activation during a working memory task. Proc. Natl. Acad. Sci. U.S.A. 97, 919-924.

van Veen, V., and Carter, C. S. (2002). The anterior cingulate as a conflict monitor: fMRI and ERP studies. Physiol. Behav. 77, 477-482.

Vertes, R. P. (2006). Interactions among the medial prefrontal cortex, hippocampus and midline thalamus in emotional and cognitive processing in the rat. Neuroscience 142, 1-20.

Vinogradova, O. S., Brazhnik, E. S., Kitchigina, V. F., and Stafekhina, V. S. (1993). Acetylcholine, theta-rhythm and activity of hippocampal neurons in the rabbit-IV: Sensory stimulation. Neuroscience 53, 993-1007.

Vogt, B. A., and Vogt, L. (2003). Cytology of human dorsal midcingulate and supplementary motor cortices. J. Chem. Neuroanat. 26, 301-309.

von Stein, A., and Sarnthein, J. (2000). Different frequencies for different scales of cortical integration: From local gamma to long range alpha/theta synchronization. Int. J. Psychophysiol. $38,301-313$.

Wang, C., Ulbert, I., Schomer, D. L., Marinkovic, K., and Halgren, E. (2005). Responses of human anterior cingulate cortex microdomains to error detection, conflict monitoring, stimulus-response mapping, familiarity, and orienting. J. Neurosci. 25 , 604-613.

Weissman, D. H., Roberts, K. C., Visscher, K.M., and Woldorff,M. G. (2006). The neural bases of momentary lapses in attention. Nat. Neurosci. 9, 971-978.

Williams, J. M., and Givens, B. (2003). Stimulation-induced reset of hippocampal theta in the freely performing rat. Hippocampus 13, 109-116.

Wilson, M. A., and McNaughton, B. L. (1993). Dynamics of the hippocampal ensemble code for space. Science 261, 1055-1058.

Conflict of Interest Statement: The authors declare that the research was conducted in the absence of any commercial or financial relationships that could be construed as a potential conflict of interest.

Received: 16 October 2010; accepted: 14 February 2011; published online: 04 March 2011.

Citation: Hyman JM, Hasselmo ME and Seamans JK (2011) What is the functional relevance of prefrontal cortex entrainment to hippocampal theta rhythms? Front. Neurosci. 5:24. doi: 10.3389/ fnins.2011.00024

Copyright ( 1011 Hyman, Hasselmo and Seamans. This is an open-access article subject to an exclusive license agreement between the authors and Frontiers Media $S A$, which permits unrestricted use, distribution, and reproduction in any medium, provided the original authors and source are credited. 